\title{
Rodriguezia bahiensis Rchb. f. : biologia floral, polinizadores e primeiro registro de polinização por moscas Acroceridae em Orchidaceae ${ }^{1}$
}

\author{
RAQUEL CARVALHO ${ }^{2,4}$ e ISABEL CRISTINA MACHADO ${ }^{3}$
}

(recebido: 9 de março de 2006; aceito: 27 de julho de 2006)

\begin{abstract}
Rodriguezia bahiensis Rchb. f.: floral biology, pollinators, and first record of Acroceridae fly pollination in Orchidaceae). Rodriguezia is distributed throughout Tropical America, comprising nearly 40 species, most of them little known regarding floral biology and pollination. Rodriguezia bahiensis is endemic to northeastern region of Brazil, it bears flower features that suggest pollination by butterflies and bees. However, the population studied is associated to a large array of visitors, including bees, butterflies, moths, hummingbirds and Acroceridae flies, the last group being for the first time recorded as pollinators in Orchidaceae. Flowers offer low quantities of nectar as reward that together with the nectar guides imitating pollen suggests the occurrence of a combined system of reward and deceit of pollinators. Though population studied is associated with a high number of visitors, natural fruit set is low (6.57\%) may be due to high frequency of pillagers and possible self-incompatibility in the studied species.
\end{abstract}

Key words - fly pollination, generalist Orchidaceae, Rodriguezia, Philopota

RESUMO - (Rodriguezia bahiensis Rchb. f.: biologia floral, polinizadores e primeiro registro de polinização por moscas Acroceridae em Orchidaceae). O gênero Rodriguezia distribui-se pela América Tropical, com cerca de 40 espécies, pouco conhecidas quanto à biologia floral e polinização. Rodriguezia bahiensis é endêmica ao Nordeste do Brasil e apresenta flores com atributos associados às síndromes de psicofilia e melitofilia. No entanto, a população estudada está associada a uma ampla guilda de visitantes florais incluindo borboletas, abelhas, mariposas, beija-flores e moscas Acroceridae, este último grupo registrado pela primeira vez atuando como polinizadores em representantes de Orchidaceae. As flores apresentam néctar como recurso, encontrado em baixa quantidade, o que em conjunto com a presença de guias de néctar imitando pólen sugere a ocorrência de um mecanismo de polinização combinando recompensa e engano. Embora a população estudada esteja associada a uma larga guilda de visitantes, a formação natural de frutos da população é baixa (6,57\%), o que pode estar associado à alta frequência de pilhadores e possível auto-incompatibilidade na espécie estudada.

Palavras-chave - Orchidaceae generalista, polinização por moscas, Rodriguezia, Philopota

\section{Introdução}

Com cerca de 25.000 espécies (Dresler 1981), a família Orchidaceae apresenta alta diversidade de visitantes e polinizadores (Dressler 1981, Endress 1994, Singer \& Cocucci 1997, Steiner 1998). Embora haja um consenso acerca da tendência à especificidade nas relações entre polinizadores e Orchidaceae (Pijl \& Dodson 1966, Proctor et al. 1996), alguns gêneros e espécies apresentam sistemas de polinização envolvendo grupos bastante diversificados de polinizadores efetivos (Dressler 1981).

\footnotetext{
1. Parte da dissertação de mestrado do primeiro autor, Programa de Pós-Graduação em Biologia Vegetal da Universidade Federal de Pernambuco.

2. Programa Amazônia - Conservação Internacional.

3. Universidade Federal de Pernambuco, Departamento de Botânica, Rua Prof. Nelson Chaves s/n, 50372-970 Recife, PE, Brasil.

4._Autor para correspondência: raquelcarvalho_brasil@hotmail.com
}

O gênero Rodriguezia, restrito à América Tropical, possui c. de 40 espécies e pertence à subtribo Oncidiinae, para a qual os registros de polinização existentes mencionam como polinizadores abelhas (Vogel 1969, Dressler 1981, Singer 2003), vespas (Singer \& Cocucci 1999) e beija-flores (Dodson 1967, Pijl \& Dodson 1966, Vogel 1969).

O néctar é um recurso floral encontrado em flores de Orchidaceae visitadas por borboletas, abelhas, beijaflores e também em algumas flores visitadas por moscas, sendo a concentração e o volume variáveis (Baker \& Baker 1982, Vogel 1983). Nas flores nectaríferas de Orchidaceae, o néctar geralmente é produzido e armazenado no cálcar, uma estrutura que surge como prolongamento de algum segmento do perianto, podendo ser tubular ou saquiforme (Dressler 1981). Alguns tipos de cálcares podem restringir a atividade dos visitantes a um único grupo com capacidade de acessar o recurso floral (Robertson \& Wyatt 1990, Johnson 1997a, b), enquanto outros podem ser acessíveis a diferentes grupos de visitantes (Smith \& Snow 1976, Patt et al. 1989, 
Okada et al. 1996).

Os Diptera representam, depois dos Hymenoptera, o mais importante grupo de polinizadores de Orchidaceae (Pijl \& Dodson 1966, Christensen 1994, Endress 1994). As flores polinizadas por moscas comumente apresentam, como aspectos atrativos, odores de frutas e cores escuras e, algumas vezes, o oferecimento de néctar, sendo comuns os mecanismos de aprisionamento de polinizadores (Dodson 1967, Christensen 1994). Dentre as Orchidaceae, as subtribos Pleurothallidinae, de distribuição neotropical, e Bulbophyllinae, de distribuição pantropical, são os dois principais grupos relacionados a esta síndrome de polinização (Christensen 1994, Borba \& Semir 1998, 2001).

No presente trabalho foram investigados a biologia floral, a polinização e o papel dos diversos visitantes florais no sucesso reprodutivo de Rodriguezia bahiensis, com base no comportamento e freqüência dos visitantes/pilhadores e polinizadores registrados para a população estudada.

\section{Material e métodos}

Local de estudo - Os estudos foram realizados na Mata do Camucim, um remanescente florestal de $c a$. 300 hectares, localizado na Estação Ecológica de Tapacurá (8² $\mathrm{S}$ e 34 ${ }^{\circ} 55^{\prime}$ W), a 52 km de Recife, Pernambuco. A altitude na área varia entre 175 a $341 \mathrm{~m}$. A vegetação predominante é do tipo floresta estacional semidecidual, havendo também áreas de mata secundária e outras desmatadas (Andrade Lima 1960, Vasconcelos Sobrinho 1970). O clima é caracterizado por duas estações bem definidas, a estação de chuvas (entre abril e agosto) e a estação seca (entre setembro e março). A precipitação média anual é de 1.284,1mm (724,4-2.105,2mm em 14 anos), com temperatura média anual de $25,6^{\circ} \mathrm{C}$ (Sudene 1990 ).

O estudo foi desenvolvido entre maio/1999 e abril/2000, perfazendo um total de 131 horas de observações de campo, sendo 114 horas diurnas, realizadas entre 6 h00 e 17h00, e 17 horas noturnas, realizadas entre $18 \mathrm{~h} 00$ e $5 \mathrm{~h} 00$, tendo sido observadas 15 plantas distribuídas em uma área de aproximadamente $100 \mathrm{~m}^{2}$.

Morfologia e biologia floral - Foram obtidos dados de morfologia floral, horário de início e seqüência da antese e de emissão de odor, produção e concentração de néctar, longevidade floral, freqüência e comportamento dos visitantes, remoção e deposição de polinários, bem como caracterização do sistema reprodutivo.

Medidas, descrição e ilustração das flores foram feitas a partir de material conservado em álcool 70\%. Para determinação do local de produção de odor foram utilizadas flores frescas inteiras ou dissecadas, coradas com vermelho neutro (Vogel 1990, Dafni 1992). Flores frescas foram também dissecadas (sépalas, pétalas, mento, labelo e coluna) e colocadas separadamente em frascos de vidro, mantidos tampados por uma hora, para determinação do local de emissão e melhor caracterização do odor (Dafni 1992).

A longevidade floral, período decorrido entre o início da antese e a senescência das flores, foi determinada para flores com polinários ( $n=6$ flores), para flores cujos polinários foram removidos ( $n=4$ flores) e para flores polinizadas ( $n=6$ flores).

O volume e a concentração de açúcares do néctar foram medidos, em flores de diferentes indivíduos, utilizando-se microseringas graduadas de $10 \mathrm{~mL}$ e refratômetro de bolso. Para a coleta desses dados, as flores $(n=8)$ foram mantidas isoladas desde a fase de botão até o quarto dia de antese, e as medições de néctar realizadas diariamente no período da manhã, entre 8h00 e $12 \mathrm{~h} 00$.

Sistema reprodutivo - Para caracterização do sistema reprodutivo foram utilizados botões isolados ou somente marcados aos quais foram aplicados quatro tratamentos: 1) emasculação e ensacamento para verificação de agamospermia ( $n=23)$; 2) autopolinização manual após antese, para verificação de autocompatibilidade $(n=11)$; 3) marcação e ensacamento de flores não manipuladas para verificação de autopolinização espontânea $(n=21)$ e 4) marcação de flores não manipuladas para controle $(n=350)$. Polinizações cruzadas manuais não foram realizadas em função do período de floração restrito e reduzida disponibilidade de flores durante o estudo.

Visitantes e taxa de remoção de polinários - Foram também registrados os visitantes florais, o horário de visita, o comportamento, a freqüência de visitas total e por hora de observação e a remoção e deposição de polinários. Os visitantes foram classificados em raros (menos de 10 visitas ao longo do período de observação), ocasionais (entre 10 e 20 visitas) e freqüentes (mais de 20 visitas).

Análises estatísticas - Para análise da freqüência total de visitas foi utilizado o teste $G$ e para a freqüência de visitas por hora de observação o teste de Wilcoxon (teste t pareado não paramétrico). Aos dados de remoção e deposição de polinários e flores abertas por dia foi aplicada a análise de regressão simples. Para análise do volume e concentração de néctar entre os dias foi utilizado o método de Kruskall Wallis (Siegel 1979, Zar 1999).

Material testemunho - Os insetos foram coletados, montados a seco e incluídos na coleção do Laboratório de Biologia Floral e Reprodutiva-UFPE. Os beija-flores foram fotografados, sendo os mesmos identificados através de literatura especializada (Grantsau 1988) e comparação com material testemunho depositado na coleção de aves do Departamento de Zoologia da UFPE. O material botânico foi incorporado ao Herbário da Universidade Federal de Pernambuco como espécime testemunho (UFP 27715).

\section{Resultados}

Hábito, fenologia da floração e morfologia floral Rodriguezia bahiensis Rchb.f. é uma espécie de hábito 
epifítico, com populações apresentando padrão de floração anual entre os meses de abril e maio de 1999 e março e maio de 2000, nas áreas estudadas.

Suas flores são hermafroditas, com dimensões de $4,18 \times 3,68 \mathrm{~cm}$, brancas com manchas roxo-claro nas porções externas das sépalas, pétalas e mento (cálcar) dispostas em inflorescências racemosas, tendo sido registradas, em média, 2,9 $\pm 1,37(n=10)$ inflorescências por planta e, em média, 10,73 \pm 4,70 $(n=19)$ flores por inflorescência. As sépalas, dorsal e laterais, são sub-lanceoladas e as pétalas lanceoladas. O labelo é fortemente trilobado, com guias de néctar no lobo médio, na forma de uma calosidade proeminente amarelo ouro, que se estende desde o ponto de inserção do labelo até a porção média deste lobo. O mento, onde se acumula o néctar, abriga o nectário floral e se localiza na base do labelo, sendo formado pelas sépalas laterais soldadas em toda sua extensão. O nectário floral é diminuto, arredondado, formado pelo prolongamento do pé da coluna. A coluna é ereta, com dois braços diminutos, abrigando o polinário e a cavidade estigmática. O polinário é simples e ereto, composto de um par de polínias amarelo-claro, diminutas, presas a um estipete elástico e a um viscídio cinzento. A cavidade estigmática é simples, localizada na porção apical da coluna, entre os dois braços (figura 1).

Biologia floral - A antese das flores de R. bahiensis ocorreu lentamente, tendo início por volta de $18 \mathrm{~h} 00$, com o afastamento das sépalas e pétalas e distensão do labelo, e encerrando-se por volta de $2 \mathrm{~h} 00$. A partir de 4h00 a maioria das flores já se encontrava completamente aberta, porém ao longo das primeiras horas da manhã algumas flores podiam ainda continuar expandindo as peças florais. A longevidade das flores de $R$. bahiensis foi de seis dias para as flores não polinizadas, mas cujos polinários foram removidos, e de oito dias para as flores que permaneceram com polinários. Flores polinizadas murcharam em 24 horas.

O odor das flores de $R$. bahiensis é pouco perceptível, semelhante ao de polpa de melancia, e sua emissão é contínua, desde a antese até a senescência, inclusive durante a noite e em flores cujos polinários

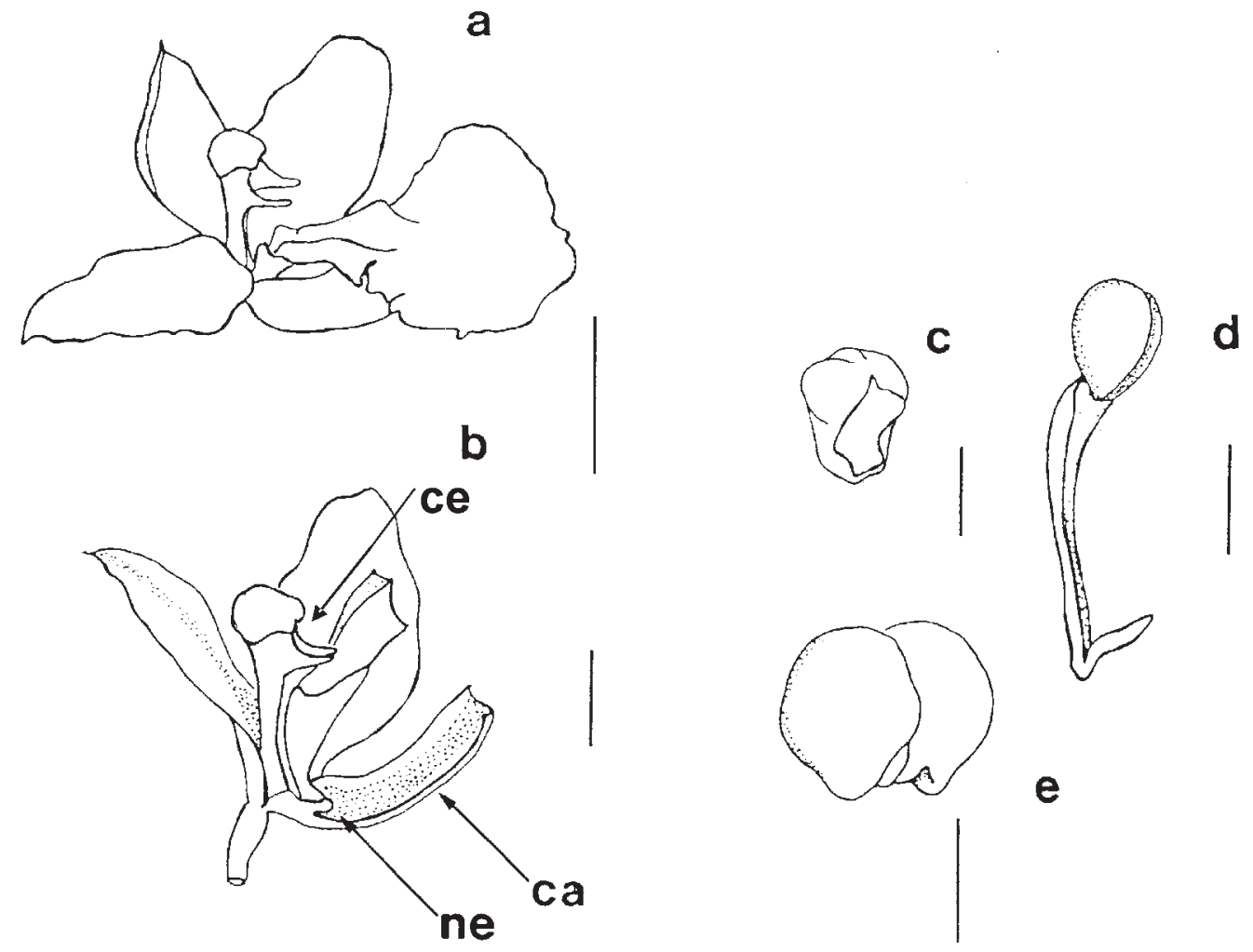

Figura 1. Flores de Rodriguezia bahiensis Rchb. f. a. Vista lateral da flor aberta. b. Vista lateral da flor dissecada mostrando coluna, nectário (ne), cavidade estigmática (ce) e cálcar (ca). c. Antera. d. Polinários. e. Estipete aderida aos polinários. Barras = $1 \mathrm{~cm}$ (a), 0,5 cm (b), 0,2 cm (c), 0,1 cm (d, e).

Figure 1. Rodriguezia bahiensis Rchb. f. flowers. a. Side view of an open flower. b. Side view of a dissected flower showing column, nectary (ne), stigmatic cavity (ce) and spur (ca). c. Anther. d. Pollinarium. e. Stipe adhered to the pollinarium. Bars = $1 \mathrm{~cm}$ (a), 0,5 cm (b), 0,2 cm (c), 0,1 cm (d, e). 
foram removidos ou que tinham sido polinizadas. O teste com vermelho neutro não indicou local específico de emissão de odor, corando apenas a região do estigma. Por sua vez, o acondicionamento das partes florais em frascos de vidro, indicou que todas as partes, exceto a coluna, emitem odor.

A produção de néctar nas flores de $R$. bahiensis iniciou-se na pré-antese e estendeu-se por um período máximo de quatro dias, sendo produzidos em média $0,4 \mu \mathrm{L}$ de néctar flor ${ }^{-1}$ dia $^{-1}$, com concentração de açúcares de 16,56\%. Entre o primeiro e o quarto dias foi registrada pequena diminuição tanto no volume quanto na concentração de açúcares, no entanto, apenas para a concentração essa redução, em cerca de 38,53\%, foi significativa $(H=6,33 ; P=0,042, n=8)$. A redução em cerca de $33,41 \%$ no volume entre os dias não foi significativa $(H=0,87 ; P=0,646, n=8)$.

Os experimentos realizados para caracterização do sistema reprodutivo indicaram que $R$. bahiensis é autoincompatível, uma vez que não houve formação de frutos nos testes de auto-polinização manual, não apresentando agamospermia nem autopolinização espontânea. Entretanto, o número de polinizações efetivadas, restringido pelo período breve de floração, impediu conclusões mais definitivas sobre o sistema reprodutivo da espécie. As flores marcadas para controle de polinização por ação dos visitantes mostraram taxa de formação natural de frutos de $6,57 \%$, ou seja, um fruto formado para cada 15,21 flores emitidas (tabela 1). Ecologia da polinização - As flores de R. bahiensis foram visitadas por borboletas (14 espécies), abelhas (cinco espécies), moscas (duas espécies), beija-flores (duas espécies), esfingídeo e noctuídeo (uma espécie cada). Dentre estes visitantes, Xylocopa (Neoxylocopa) suspecta, Philopota sp. 1 e Philopota sp. 2 (Acroceridae) atuaram como polinizadores efetivos de $R$. bahiensis (tabela 2).

Tabela 1. Sistema reprodutivo de Rodriguezia bahiensis Rchb. f. ( $n=14$ plantas).

Table 1. Reproductive system of Rodriguezia bahiensis Rchb. f. ( $n=14$ plants).

\begin{tabular}{lcc}
\hline Tratamento & $n$ & Frutos (\%) \\
\hline Controle geral* $^{*}$ & 350 & 6,57 \\
Agamospermia & 23 & 0 \\
Autopolinização espontânea & 21 & 0 \\
Autopolinização manual & 11 & 0 \\
\hline
\end{tabular}

*Floração de 2000
Tabela 2. Visitantes e polinizadores de Rodriguezia bahiensis Rchb. f. na Estação Ecológica de Tapacurá, em Pernambuco. (Raros $=<10$ visitas; Ocasionais $=10$ a 20 visitas; Freqüentes $=>20$ visitas; $\mathrm{PE}=$ Polinizador Efetivo; $\mathrm{PP}=$ Possível Polinizador; $\mathrm{PI}=$ Pilhador $)$.

Table 2. Visitors and pollinators of Rodriguezia bahiensis Rchb. f. in Estação Ecológica de Tapacurá, Pernambuco state. (Rare $=<10$ visits; Occasional $=10$ to 20 visits; Frequent $=>20$ visits; $\mathrm{PE}=$ Effective pollinator; $\mathrm{PP}=$ Possible pollinator; $\mathrm{PI}=$ Pillage).

Espécie

Atividade Freqüência

TROCHILIDAE

Amazilia fimbriata (Gmelin, 1788)

Phaethornis ruber (Linnaeus, 1758) PP

HYMENOPTERA

Euglossa cf. cordata (Linnaeus, 1758) P

Eulaema bombiformis (Friese, 1899) PI

Eulaema nigrita Lepeletier, $1841 \quad$ PI

Exaerete cf. smaragdina (Guerin, PI 1845)

Xylocopa (Neoxylocopa) suspecta PE

Moure \& Camargo, 1988

DIPTERA/ACROCERIDAE

Philopota sp. 1

Philopota sp. 2

Lepidoptera/Hesperiidae

Carystus phorcus (Cramer, 1777) P

Cobalopsis nero (Herrich-Schäffer, PI 1869)

Cobalopsis cf. miaba (Schaus, 1902)

Epargyreus socus Hübner, 1925

Nyctelius nyctelius (Latreille, 1824)

Panoquina lucas (Fabricius, 1793)

Sodalia sp. 1

Sodalia sp. 2

Spathilepia clonius (Gramer, 1775)

Urbanus esmeraldus (Butler, 1877)

Urbanus velinus (Plötz, 1881)

Indeterminado 1

Indeterminado 2

Indeterminado 3

Indeterminado 4

Indeterminado 5

Indeterminado 6

LEPIDOPTERA/PAPILIONOIDEAE

Parides zacynthus polymetus

(Godart, 1819)

LEPIDOPTERA/SPHINGIDAE

Agrius cingulata (Fabricius, 1775) PI Raro

LEPIDOPTERA/NOCTUIDAE

Indeterminado 1
Freqüente

Freqüente

Raro

Raro

Raro

Raro

Ocasional

Raro

PE Raro

Ocasional

Freqüente

Raro

Raro

Raro

Raro

Freqüente

Ocasional

Raro

Freqüente

Freqüente

Raro

Raro

Raro

Raro

Raro

Raro

Freqüente

Raro 
O comportamento de visitas registrado para as espécies de borboletas foi similar. Os visitantes abordavam as flores frontalmente, pousando sobre o labelo e inserindo o aparelho bucal entre a coluna e o labelo para obtenção de néctar. Ao se alimentarem, as borboletas permaneciam com o corpo afastado da coluna, não realizando remoção e/ou deposição de polinários. Em geral esses insetos abordavam no máximo duas flores por planta, em visitas que demoravam cerca de 2 a 3 minutos.

A freqüência de visitas total de borboletas, analisada para as espécies com freqüência superior a oito visitas, foi significativamente diferente entre as espécies ( $g=214 ; g l=14 ; P<0,0001)$, exceto entre as duas espécies de Urbanus e Cobalopsis nero $(g=0 ; g l=1$; $P=1$ ) e entre Sodalia sp. 2 e Panochyna lucas. As maiores freqüências absolutas foram registradas para Parydes zacynthus polymentus (55 visitas), Cobalopsis Nero (figura 2) (37), Urbanus esmeraldus (31), Sodalia sp. 2 (21), Sodalia sp. 1 (15) e Panochyna sp. (oito).

O esfingídeo Agrius cingulata (figura 3) e o noctuídeo não identificado foram registrados respectivamente uma e duas vezes cada durante os períodos de observação noturna, visitando uma ou duas flores por planta, não tendo sido registradas remoção ou deposição de polinários.

Euglossa cordata, Exaerete cf. smaragdina, Eulaema bombiformis e E. nigrita foram visitantes raros. Estas abelhas realizaram visitas rápidas (< de 30 segundos), em geral a uma única flor por planta ou a uma ou duas flores de uma única inflorescência, não tendo sido registradas remoção ou deposição de polinários.

Xylocopa (Neoxylocopa) suspecta sobrevoava as inflorescências, abordava as flores frontalmente, pousava, agarrava-se com as pernas anteriores à região dorsal inferior da coluna e, com as pernas medianas, ao labelo. Inseria o aparelho bucal entre a coluna e o labelo, tocando facilmente o viscídio e removendo os polinários (figura 4). As visitas de $X$. (Neoxylocopa) suspecta foram também muito rápidas (< de 30 segundos), e em geral essas abelhas visitavam todas as flores abertas de uma mesma inflorescência, removendo grande quantidade de polinários, que aderiram à região entre os alveólos antenais. Embora houvesse perda de polinários, sua remoção em grandes quantidades facilitou a deposição na cavidade estigmática das flores visitadas posteriormente.

Phaethornis ruber (figura 5) e Amazilia fimbriata abordavam as flores frontalmente, realizando visitas legítimas rápidas (< de 30 segundos), em duas ou três flores por inflorescência, no sentido da base para o ápice. Comumente também visitavam botões em préantese, inserindo o bico diretamente no mento. A remoção de polinários só foi registrada para Phaethornis ruber, embora as duas espécies tenham apresentado comportamento de visitas similar. A deposição de polinários, porém, não foi registrada para nenhuma das duas espécies de beija-flores. A freqüência de visitas total registrada para Phaethornis ruber (27 visitas) e Amazilia fimbriata (28 visitas) não foi significativamente diferente $(g=0 ; g l=1 ; P=1)$, nem a freqüência de visitas por hora de observação $(z=1$; $P=1)$.

As duas espécies de Acroceridae, Philopota sp. 1 e Philopota sp. 2, abordavam as flores frontalmente, inserindo o aparelho bucal entre a coluna e o labelo, permanecendo longo tempo (mais de 3 minutos) visitando uma única flor, facilmente tocando o viscídio e removendo os polinários, que aderiam fortemente ao ápice do dorso destas moscas, próximo à cabeça (figuras 6 e 7). A deposição, facilitada pelo maior tempo de permanência dessas moscas nas flores, ocorreu pelo contato dos polinários com a cavidade estigmatífera. As menores perdas de polinários podem ser atribuídas a estes polinizadores. As moscas visitaram comumente apenas uma flor por inflorescência.

A freqüência total de visitas foi significativamente diferente entre todos os polinizadores $(g=21 ; g l=3$; $P<0,0001$ ). Phaethornis ruber (27 visitas) foi o polinizador mais freqüente, seguido por Xylocopa (Neoxylocopa) suspecta (10), Philopota sp. 1 (sete) e Philopota sp. 2 (uma). A freqüência de visitas por hora de observação foi significativamente diferente apenas com relação a Philopota sp. $2(P<0,0001)$.

A taxa de remoção de polinários foi de $30,27 \%$ e a de deposição foi de 20,18\%, para 109 flores examinadas. De acordo com a análise de regressão linear, tanto o número de polinários removidos $(f=32 ; P<0,0001)$, quanto o número de polinários depositados $(f=41$; $P<0,0001)$, aumentaram em função do número de flores abertas por dia.

\section{Discussão}

Embora a morfologia e os atributos florais no gênero Rodriguezia indiquem a ocorrência predominante das síndromes de psicofilia e ornitofilia (Dodson 1967, Pijl \& Dodson 1966, Vogel 1969, Dressler 1981), os dados do presente trabalho sugerem que as espécies deste gênero possam estar associadas a guildas mais 


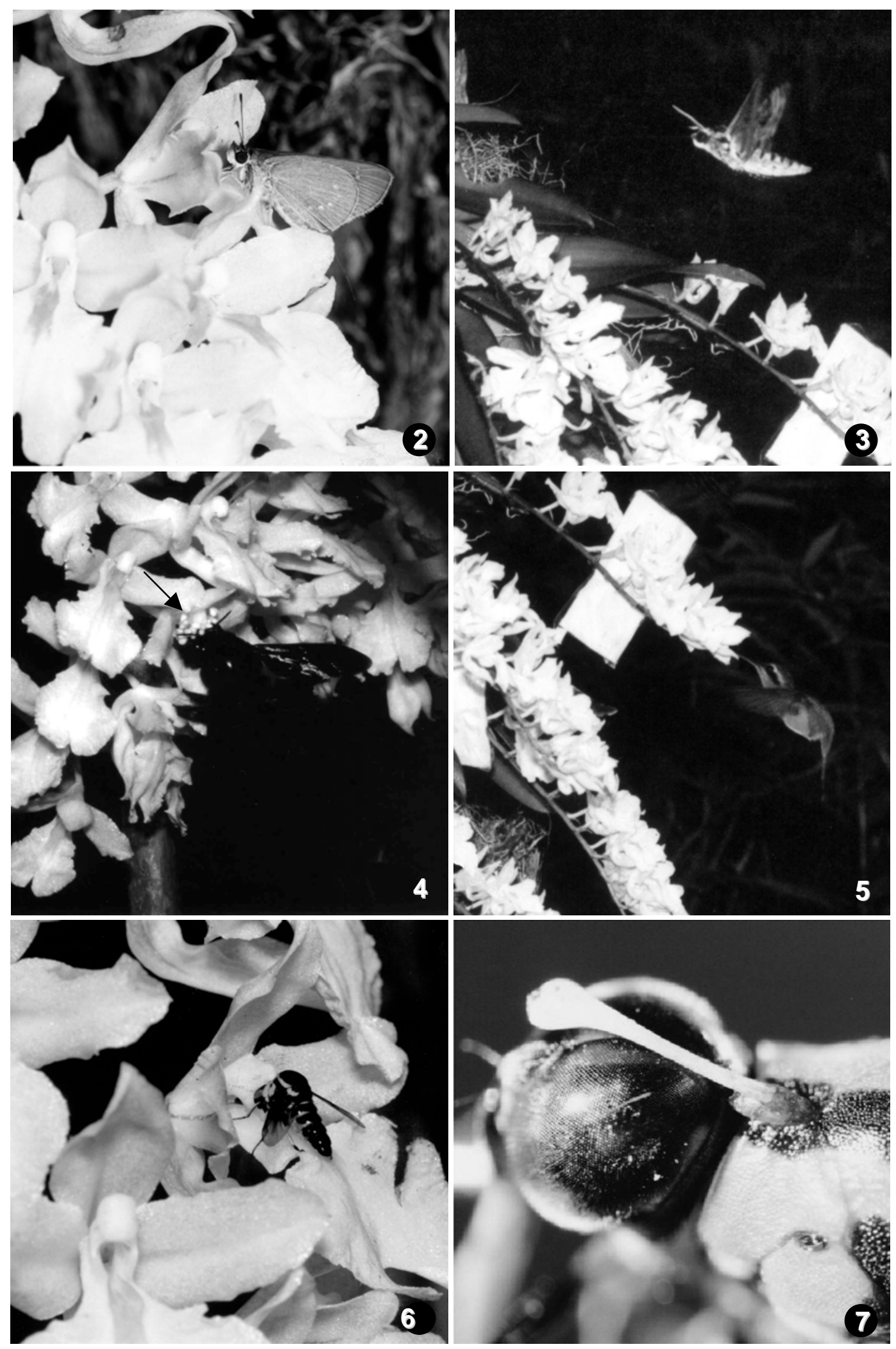

Figuras 2-7. Visitantes (2, 3, 4) e polinizadores $(5,6,7)$ de Rodriguezia bahiensis Rchb. f. 2. Cobalopsis nero (Hesperiidae). 3. Agrius cingulata (Sphingidae). 4. Adesão de polinários na cabeça (seta) de Xylocopa (Neoxylocopa) suspecta (Apidae). 5. Phaethornis ruber (Trochilidae). 6. Philopota sp. 1 (Acroceridae) tocando coluna da flor de R. bahiensis. 7. Polinário aderido no dorso de Philopota sp. 1 (Acroceridae). 
diversificadas de visitantes e polinizadores. Rodriguezia bahiensis Rchb. f., em particular, apresenta características florais que dificultam seu enquadramento em alguma das síndromes florais (sensu Faegri \& Pijl 1979) e, efetivamente, a diversidade de visitantes aponta para isto. É importante ressaltar, no entanto, que $R$. bahiensis é a primeira espécie do gênero a ser estudada em detalhe e maiores informações acerca de outras espécies do grupo são necessárias para compreender efetivamente quão diversos podem ser os visitantes e polinizadores associados a este gênero.

O cálcar, que abriga o nectário floral de $R$. bahiensis, não sendo um tubo longo como encontrado em flores ornitófilas, psicófilas e esfingófilas, e sim uma estrutura aberta e de pouca profundidade, favoreceu mais a polinização por moscas e abelhas de língua curta. Nas flores de $R$. bahiensis, enquanto as várias espécies de visitantes de aparelho bucal longo (lepidópteros e abelhas Euglossa e Exaerete) permaneciam com o corpo afastado da coluna, sem remover ou depositar polinários, os visitantes de aparelho bucal relativamente mais curto (moscas Acroceridae e abelhas Xylocopa), ao contrário, forçavam a coluna com a cabeça ou com o dorso para obtenção de néctar, possibilitando a remoção e posterior deposição de polinários.

Assim como para as espécies de borboletas, a atividade das duas espécies de lepidópteros noturnos possivelmente foi de pilhagem. A polinização por esfingídeos em Orchidaceae tem sido relacionada principalmente às espécies de Habenaria e Angraecum (Dodson 1967, Dressler 1981), as quais geralmente apresentam cálcares bastante longos.

O beija-flor Phaethornis ruber, freqüentemente observado como visitante de $R$. bahiensis, é reconhecidamente de comportamento oportunista, visitando espécies de diferentes tipos florais, nem sempre com atributos ornitófilos (Siqueira \& Machado 1998, Machado \& Lopes 2000). Esta espécie, embora tenha removido polinários, não foi observada depositando-os, sendo, desta forma, considerada apenas como um possível polinizador da espécie estudada.

Para as Orchidaceae, de acordo com Christensen (1994) e Proctor et al. (1996), as moscas são o segundo grupo mais importante de polinizadores depois das abelhas, sendo a família Syrphidae uma das mais importantes como polinizadores dentre as moscas. Em Orchidaceae, a polinização por moscas predomina em gêneros como Pleurothallis e Bulbophyllum, e envolve, como aspectos atrativos, flores com odores de frutas ou putrescentes, cores escuras, mimetismo de locais para oviposição, mecanismos de aprisionamento de vetores e, algumas vezes, o oferecimento de néctar (Sazima 1978, Endress 1994, Christensen 1994, Borba \& Semir 1998, 2001). Estas características, com exceção do néctar, não são encontradas nas flores de $R$. bahiensis, o que torna ainda mais singular o presente registro de polinização por moscas. Mais singular ainda é o registro de Philopota (Acroceridae) como polinizadores. Os Acroceridae constituem um pequeno grupo de moscas pouco estudado com relação à sua biologia, especialmente na Região Neotropical (Luz 2004). Algumas de suas espécies, pertencentes à subfamília Philopotinae, podem atuar como polinizadores, enquanto se alimentam de néctar (Luz 2004), como é o caso das duas espécies de Philopota registradas pela primeira vez neste trabalho em associação com $R$. bahiensis. Até o momento, não havia sido registrada a visita e a polinização por representantes de Acroceridae em quaisquer espécies de Orchidaceae.

A deposição dos polinários em $R$. bahiensis pelas espécies de Acroceridae registradas pode ser considerada como mais eficiente em relação às outras espécies de polinizadores pois, embora a freqüência de visitas e o número de polinários removidos tenham sido menores, o contato dos polinários com a cavidade estigmática era mais prolongado nas moscas do que para as demais espécies, aumentando as chances de deposição dos polinários. Além disso, as duas espécies de Acroceridae registradas apresentam a porção dorsal apical do tórax proeminente, formando um escudo característico do gênero Philopota (Luz 2004), o que também auxiliou a remoção e deposição dos polinários.

A taxa de formação natural de frutos de 6,57\%, registrada para $R$. bahiensis, é inferior ao citado para outras espécies nectaríferas de Orchidaceae, exceto para Arethusa bulbosa L., com formação natural de frutos de 5\% (Thien \& Marcks 1972). Em geral, os níveis de frutificação registrados para outras espécies de Orchidaceae oscilam entre $16 \%$ e 52\% (Thien \& Marcks 1972, Smith \& Snow 1976, Patt et al. 1989),

Figures 2-7. Visitors (2, 3, 4) and pollinators (5, 6, 7) of Rodriguezia bahiensis Rchb. f. 2. Cobalopsis nero (Hesperiidae). 3. Agrius cingulata (Sphingidae). 4. Xylocopa (Neoxylocopa) suspecta (Apidae) with pollinarium on the head (arrow). 5. Phaethornis ruber (Trochilidae). 6. Philopota sp. 1 (Acroceridae) touching the column of a R. bahiensis flower. 7. Philopota sp. 1 (Acroceridae) with pollinarium. 
havendo valores superiores em espécies com populações apomíticas, como Pogonia ophioglossoides (L.) Ker Gawl. (Thien \& Marcks 1972).

A maioria das Orchidaceae nectaríferas de regiões temperadas e subtropicais apresenta taxa natural de frutificação superior a 50\%, ao contrário do registrado para regiões tropicais, onde as taxas de frutificação são baixas. Segundo Neiland \& Wilcock (1998) este fato pode ser atribuído principalmente à falta de polinizadores eficientes. Para $R$. bahiensis, dentre as 26 espécies de visitantes florais, apenas duas moscas e Xylocopa suspecta (Apidae) atuaram efetivamente como polinizadores, devido ao comportamento de visita e também pelas taxas de remoção e deposição de polinários. Os demais visitantes atuaram como possíveis polinizadores ou como pilhadores de néctar. Alguns desses pilhadores, como as borboletas, apresentaram freqüência de visitas total muito superior à dos polinizadores.

Os resultados dos experimentos de polinização manual, sugerindo auto-incompatibilidade, associados à baixa freqüência dos polinizadores efetivos e à interferência de pilhadores reduzindo a disponibilidade de néctar poderiam explicar as baixas taxas de formação de frutos na espécie.

Embora a família Orchidaceae seja descrita como um grupo bastante específico quanto à atividade de visitantes e polinizadores, não são raros os casos de gêneros e espécies com sistemas de polinização diversificados e generalistas. O gênero Satyrium, ocorrente na África do Sul, apresenta espécies polinizadas por borboletas, mariposas, abelhas e moscas, havendo em S. ligulatum sobreposição de atividade entre mariposas e borboletas (Johnson 1997b). Neuwiedia veratrifolia Blume, pertencente à subfamília Apostasioideae, um dos grupos basais de Orchidaceae, foi estudada por Okada et al. (1996) em Sumatra, na Indonésia, tendo como visitantes tisanópteros, himenópteros e besouros, sendo abelhas do gênero Trigona os polinizadores efetivos. Segundo Tremblay (1992), Epipactis palustris Crantz é a espécie, dentre as Orchidaceae estudadas, que possui a maior guilda de visitantes florais, com 103 espécies de polinizadores. Para outra espécie generalista de Orchidaceae, Plathantera stricta Lindley, cujos visitantes e polinizadores podem ser espécies de dípteros, besouros, abelhas, borboletas e mariposas, esta amplitude de vetores de pólen possibilitou sua reprodução e estabelecimento em áreas com clima e disponibilidade de polinizadores imprevisíveis (Patt et al. 1989).
Abelhas Euglossa (E. nigropilosa) e borboletas Heliconius ( $H$. hermathena) registradas respectivamente por Pijl \& Dodson (1966) e Braga (1977) como polinizadores de Rodriguezia leeana Rchb. f. e $R$. secunda H.B. \& K., também são exemplos da diversidade de polinizadores relacionada ao gênero Rodriguezia.

Waser et al. (1996) e Johnson \& Steiner (2000) salientam que a dicotomia entre generalização e especialização é uma abordagem simplista, pois o que existe de fato são contínuos entre espécies polinizadas por centenas de vetores e outras, como algumas Orchidaceae, polinizadas por um único vetor. Tremblay (1992) verificou que, dentre as 479 espécies de Orchidaceae registradas quanto ao número de polinizadores, apenas cerca de 25 espécies apresentam mais de quatro polinizadores, enquanto mais de 300 espécies apresentam um único polinizador. Já Waser et al. (1996), analisando os números de polinizadores registrados para Orchidaceae da Europa, constataram que cada espécie é visitada em média por cerca de oito espécies e cinco gêneros distintos de visitantes, valores que pouco diferem do registrado para outras famílias como Ranunculaceae e Polemoniaceae.

Do ponto de vista da diversidade de polinizadores, Waser et al. (1996) salientam que não apenas o número de polinizadores, mas também a diversidade dos grupos de vetores deve ser considerada ao caracterizar uma espécie como generalista ou especialista. Os três polinizadores efetivos de $R$. bahiensis pertencem a dois grupos de insetos, quais sejam abelhas e moscas. Considerando a eficiência dos polinizadores, medida através das taxas de remoção e deposição de polinários podemos afirmar, com base na freqüência de visitas e no comportamento dos polinizadores de $R$. bahiensis, que suas contribuições à reprodução da população estudada são comparáveis, pois as menores perdas de polinários, após a remoção, foram atribuídas às duas espécies de Acroceridae, com baixa freqüência de visitas e as maiores perdas, atribuídas a polinizadores mais freqüentes, como por exemplo Xylocopa (Neoxylocopa) suspecta.

Considerando a diversidade de grupos e a eficiência dos polinizadores, como os aspectos principais a serem considerados ao se discutir o enquadramento de uma dada espécie como generalista ou especialista (Johnson \& Steiner 2000), pode-se sugerir que, apesar da diversidade de visitantes/ pilhadores, $R$. bahiensis apresenta relativa especialização no mecanismo de polinização, o qual é efetivado por apenas três espécies. 
Agradecimentos - À Capes e ao CNPq pelo apoio financeiro; Administração da Estação Ecológica do Tapacurá pelo apoio logístico; ao Missouri Botanical Garden por permitir o acesso à biblioteca desta instituição; ao Dr. Leonardo Félix (UFPB) pela identificação da espécie de Rodriguezia, ao Dr. Gustavo Acácio (USP) pela identificação das borboletas, ao Dr. Marcelo Tabarelli (UFPE) pelas sugestões nas análises estatísticas, ao Dr. Clemens Schlindwein (UFPE) pela identificação da espécie de Xylocopa; ao Museu Nacional do Rio de Janeiro pela identificação das moscas e a Alberto Vicentini pela ajuda nas observações de campo. Gostaríamos também de agradecer aos Drs. Eduardo Borba (UFMG), João Semir (Unicamp), Paulo Oliveira (UFU) e assessor anônimo, cujos comentários e sugestões em muito contribuíram para o enriquecimento de nosso trabalho.

\section{Referências bibliográficas}

ANDRADE LIMA, D. 1960. Estudos fitogeográficos de Pernambuco. Arquivos do IPA 5: 305-341.

BAKER, H.G. \& BAKER, I. 1982. Floral nectar sugar constituents in relation to pollinator type. In Handbook of experimental pollination biology (C.E. Jones \& R.J. Little, eds.). Van Nostrand, New York, p.117-141.

BORBA, E.L. \& SEMIR, J. 1998. Wind-assisted fly pollination in three Bulbophyllum (Orchidaceae) species occurring in the Brazilian Campos Rupestres. Lindleyana 13:203-218.

BORBA, E.L. \& SEMIR, J. 2001. Pollinator specificity and convergence in fly-pollinated Pleurothallis (Orchidaceae) species: a multiple population approach. Annals of Botany 88:75-88.

BRAGA, P.I.S. 1977. Aspectos biológicos das Orchidaceae de uma campina na Amazônia Central. Acta Amazonica 7 (suplemento 2):1-89.

CHRISTENSEN, D.E. 1994. Fly pollination in the Orchidaceae. In Orchid biology: reviews and perspectives, VI. (J. Arditti, ed.). John Wiley and Sons, London, p.415-449.

DAFNI, A. 1992. Pollination ecology. A practical approach. Oxford University Press, Oxford.

DODSON, C.H. 1967. Relationships between pollinators and orchid flowers. Atas do Simpósio sobre a Biota Amazônica 5:1-72

DRESSLER, R.L. 1981. The orchids. Natural History and classification. Harvard University Press, Cambridge.

ENDRESS, P.K. 1994. Diversity and evolutionary biology of tropical flowers. Cambridge University Press, Cambridge.

FAEGRI, K. \& PIJL, L.VAN DER. 1979. The principles of pollination ecology. Pergamon Press, Oxford.

GRANTSAU, R. 1988. Die Kolibris Brasiliens. Expressão e Cultura, Rio de Janeiro.

JOHNSON, S.D. 1997a. Pollination ecotypes of Satyrium hallackii (Orchidaceae) in South Africa. Botanical Journal of the Linnean Society 123:225-235.
JOHNSON, S.D. 1997b. Insect pollination and floral mechanisms in South African species of Satyrium (Orchidaceae). Plant Systematics and Evolution 204:195-206.

JOHNSON, S.D. \& STEINER, E. 2000. Generalization versus specialization in plant pollination systems. Trends in Ecology and Evolution 15:140-143.

LUZ, J.R.P. 2004. A associação de Philopota sp. Wiedemann (Diptera, Acroceridae) com flores do Gervão-Azul, Stachytarphetta cayensis (Verbenaceae) na Ilha de Marambaia, Rio de Janeiro, Brasil. Entomología y Vectores 11:681-687.

MACHADO, I.C. \& LOPES, A.V.F. 2000. Souroubea guianensis: quest for its pollinators and the first case of tapetal oil in Marcgraviaceae. Annals of Botany 85:705-711.

NEILAND, M.R. \& WILCOCK, C.C. 1998. Fruit set, nectar reward and rarity in the Orchidaceae. American Journal of Botany 85:1657-1671.

OKADA, H., KUBO, S. \& MORI, Y. 1996. Pollination system of Neuwiedia veratrifolia Blume (Orchidaceae, Apostasioideae) in the Malesian Wet Tropics. Acta Phytotaxonomica et Geobotanica. 47:173-181.

PATT, J.M., MERCHANT, M.W., WILLIMAS, D.R.E. \& MEUSE, B.J. 1989. Pollination biology of Platanthera stricta (Orchidaceae) in Olympic National Park, Washington. American Journal of Botany 76:1098-1105.

PIJL, L. VAN DER \& DODSON, C.H. 1966. Orchid flowers: their pollination and evolution. University of Miami Press, Coral Gables.

PROCTOR, J.L., YEO, P. \& LACK, A. 1996. The natural history of pollination. Timber Press, Portland.

ROBERTSON, J.L. \& WYATT, R. 1990. Reproductive biology of the yellow fringued orchid, Platanthera ciliaris. American Journal of Botany 77:388-398.

SAZIMA, M. 1978. Polinização por moscas em Bulbophyllum warmingianum Cogn. (Orchidaceae), na Serra do Cipó, Minas Gerais, Brasil. Revista Brasileira de Botânica 1:133-138.

SIEGEL, S. 1979. Estatística não paramétrica: para as ciências do comportamento. McGrawHill do Brasil, Rio de Janeiro.

SINGER, R.B. 2003. Orchid pollination: recent developments from Brazil. Lankesteriana 7:111-114.

SINGER, R.B. \& COCUCCI, A.A. 1997. Pollination of Pteroglossaspis ruwenzoriensis (Rendle) Rolfe (Orchidaceae) by beetles in Argentina. Botanica Acta 110:338-342.

SINGER, R.B. \& COCUCCI,A.A. 1999. Pollination mechanisms in four sympatric southern Brazilian Epidendroideae orchids. Lindleyana 14:47-56.

SIQUEIRA, J.A. \& MACHADO, I.C. 1998. Biologia floral de Hohenbergia ridleyi (Baker) Mez. Bromelia 5:3-13. 
SMITH, G.R. \& SNOW, G.E. 1976. Pollination ecology of Plathantera ciliaris and $P$. blephariglottis (Orchidaceae). Botanical Gazette 13:133-140.

STEINER, K.E. 1998. The evolution of beetle pollination in a south african orchid. American Journal of Botany 85:1180-1193.

SUDENE. 1990. Dados pluviométricos do Nordeste - Estado de Pernambuco. Série Pluviométrica 6. Superintendência do Desenvolvimento do Nordeste, Recife.

THIEN, L.B. \& MARCKS, B.G. 1972. The floral biology of Arethusa bulbosa, Calopogon tuberosus and Pogonia ophioglossoides (Orchidaceae). Canadian Journal of Botany 50:2319-2325.

TREMBLAY, R.L. 1992. Trends in the pollination ecology of the Orchidaceae: evolution and systematics. Canadian Journal of Botany 70:642-650.
VASCONCELOS SOBRINHO, J. 1970. As regiões naturais do Nordeste, o meio e a civilização. Companhia Editora de Pernambuco, Recife.

VOGEL, S. 1969. Über synorganisierte Blütensporne bei einigen Orchideen. Österreichische Botanische Zeitschrift 116: 244-262.

VOGEL, S. 1983. Ecophysiology of zoophilic pollination. In Physiological plant ecology III. (O. L. Lange, P.S. Nobel, C.B. Osmond \& H. Ziegler, eds.). Berlin, p.560-611.

VOGEL, S. 1990. The role of scent glands in pollination (Translated by S.S. Renner). Smithsonian Institution. Washington, D.C.

WASER, N.M., CHITTKA, L., PRICE, M.V., WILLIAMS, N.M. \& OLLERTON, J. 1996. Generalization in pollination systems, and why it matters. Ecology 77:1043-1060.

ZAR, J.H. 1999. Biostatistical analysis. 4th ed. Prentice Hall, Upper Saddle River. 